\title{
Epidemiology and Treatment Outcomes of Sinonasal Tumors: A Single Institute's Experience in Pakistan
}

\author{
Wajiha Anjum1, Rab Nawaz Maken1, Hasan Nisar3, Ismat Fatima², Misbah Masood1 and Abu Baker Shahid1
}

\begin{abstract}
Objective: To find the epidemiology and risk factors of sinonasal tumors and treatment outcomes in squamous cell carcinoma.

Study Design: An observational study.

Place and Duration of Study: The Institute of Nuclear Medicine and Oncology Lahore (INMOL), Lahore, from May 2016 to March 2017.

Methodology: All histopathologically proven cases of paranasal sinuses and nasal cavity were selected from the hospital record for epidemiological analysis. Survival outcomes of patients with squamous cell histopathology were determined, which is commonly occurring type. Relevant information was obtained from patient record and telephone communication. The data were analysed using SPSS V.20.

Results: Sinonasal malignancies are rare, making ( $n=81) 0.2 \%$ of all registered tumors; out of which, $46(56.7 \%)$ had squamous cell histology. Median age was 50.0 years (IQR: 60.7-40.0) with male predominance (1.7:1). Most of patients presented at advanced stage, T3/ T4 in more than two-thirds of cases, and associated with nodal metastasis in $43.5 \%$ of squamous cell carcinoma. In patients with squamous cell histology, median disease-free survival was 19.00 months (SE: $1.65,95 \% \mathrm{Cl}, 15.75$ - 22.25), median overall survival remained 34.00 months (SE: 1.84, 95\% Cl, $30.00-38.00$ ). Nodal status had significant effect $(p<0.001)$ on survival. Radiotherapy had significant effect on improved survival $(p=0.001)$ and distant metastasis remained negative prognostic factor $(p=0.001)$. Disease stage was also significantly associated with overall survival (Log Rank 0.014). Tumour size, surgery, chemotherapy, and chemoradiotherapy were not significantly associated with survival. Cumulative survival at 1,2 , and 3 years was $87 \%, 48 \%$ and $29 \%$, respectively.

Conclusion: Sinonasal malignancies are rare, advanced stage is common, and radiotherapy improves overall survival.
\end{abstract}

Key Words: Sinonasal malignancies, Epidemiology, Treatment outcomes.

\section{INTRODUCTION}

Sinonasal malignancies (SNMs) are a rare group of tumors with reported incidence of 1 per 100,000 people annually. They account for only $3 \%$ of upper respiratory tract tumors and $5 \%$ of all head and neck cancers. 1,2 Incidence is higher in Asia and Africa than in the United States and Europe. In parts of Asia such as Japan, SNM are the second most common subgroup of head and neck tumors preceded only by nasopharyngeal carcinoma. ${ }^{3}$

SNMs include tumors arising from nasal cavity and paranasal sinuses. Maxillary sinus is the commonest site followed by nasal cavity. Cancers of the ethmoid, frontal, and sphenoid sinuses are extremely rare. The

\footnotetext{
1 Department of Radiotherapy / Clinical Pathology2, Institute of Nuclear Medicine and Oncology Lahore (INMOL), Lahore, Pakistan

3 Department of Medical Sciences, Pakistan Institute of Engineering and Applied Sciences, (PIEAS), Islamabad, Pakistan

Correspondence: Dr. Ismat Fatima, Department of Clinical Pathology, Institute of Nuclear Medicine and Oncology Lahore (INMOL), Lahore, Pakistan

E-mail: drismatfatima14@gmail.com

Received: May 18, 2018; Accepted: October 31, 2018
}

annual incidence of maxillary sinus cancer is $0.5-1.0$ case per 100,000 of the population. The disease has a predilection for males with male to female ratio being 2.3:1.4 The exact site of origin is difficult to determine because these tumors mostly present at an advanced stage and extensively involve adjacent sites. Most patients with carcinomas arising in the sinonasal region are more than 40 years of age. Lymph node metastases are not frequently found unlike squamous cell tumors in other sites of the head and neck region, with incidence ranging from $3.3 \%$ to $26 \%$. The most common histology is squamous cell carcinoma; other varieties being adenoid cystic carcinoma, adenocarcinoma, olfactory neuroblastoma, plasmacytoma, lymphoma, malignant melanoma, and sarcoma.5,6 These tumors are often reported late and may be misdiagnosed initially due to nonspecific nature of early symptoms that may include nasal congestion or blockage, blood-stained discharge and anosmia. Subsequent extension into the orbit, palate, skin, infra temporal fossa, cavernous sinus or the pterygo-maxillary fissure, may produce symptoms such as proptosis, non-healing skin ulcer, trismus or other mass effects or neurological deficits.

In the early stage, tumour resection may be entirely endoscopic but early stage disease, as explained earlier, is uncommon and surgical excision procedures mostly include craniofacial resection, maxillectomy (mid-facial 
degloving and lateral rhinotomy) and orbital exenteration. Given the complicated anatomy of the region, negative margin surgery is quite a challenge in locally advanced cases. This makes adjuvant radiotherapy a useful option in such cases. However, irradiation to a radical dose is itself a challenge due to close proximity to many organs at risk of damage from radiation such as lens, retina, optic nerve, and chiasm and pituitary gland.

The rationale of the present retrospective review of record was the scarcity of relevant local data with regard to its epidemiological details and treatment outcomes in the backdrop of relatively higher incidence of SNM in Asia.

The objective of this study was to determine the common epidemiological features of patients with SNM and analyse treatment outcomes of SNM squamous cell carcinoma (SCC) patients presenting INMOL, using a 15-year data (2001-2015).

\section{METHODOLOGY}

This observational study was conducted at INMOL, Lahore, from May 2016 to March 2017. Selection criteria comprised of all histopathologically proven cases of paranasal sinuses and nasal cavity that had been treated at INMOL, Lahore, from January 2001 to December 2015. Data were collected from the patients' medical records and contact was established via telephone, where necessary. Non-probability purposive sampling was done for the purpose.

Data collection was done on an SPSS version 20 sheet, designed to contain all study variables which were both epidemiology based and treatment outcome related. These included gender, median age, histopathology, primary tumor site, stage, nodal status, presenting symptoms, risk factors, median overall survival (OS), and disease-free survival (DFS). TNM staging system was used for the purpose of our study which was relied on AJCC Cancer Staging Manual, 2010. Radiation doses were either 60 Gy in case of a clear margin surgery or $66 \mathrm{~Gy}$ in case of close or positive margins surgery using conventional fractionation. CCRT was reserved for cases which were deemed inoperable in a Tumor Board discussion either for medical reasons or due to wide extent of disease rendering it inoperable. All patients who received CCRT were given cisplatin at 40 $\mathrm{mg} / \mathrm{m}^{2}$ at weekly intervals along with $60 \mathrm{~Gy}$ of radiation using conventional fractionation scheme. All patients, except stage IVC and an ECOG performance status of 2 or under, were treated with a curative intent.

Ethical approval was obtained from the Institutional Review Board prior to the study. For data analysis, SPSS version 20.0 was used. The tests of significance applied were Chi-square and Log-rank test, p-value $<0.05$ was considered significant. Median DFS and OS were calculated using Kaplan-Meier statistics.

\section{RESULTS}

Eighty-one patients were included in the study. Median age of the study population was 50.00 years (IQR: $60.75-40.00$ ). Male and female patients were $62 \%$ $(n=51)$ and $38 \%(n=30)$, respectively, male to female ratio being 1.7:1.0 (Table I). Squamous cell carcinoma (SCC) was the most common histopathology (56.7\%, $\mathrm{n}=46$ ). Maxillary sinus was the most common primary site $(50.6 \%, \mathrm{n}=41)$. Most frequent presenting symptom was nasal blockage and headache, while smoking was the major risk factor (Table I).

Pretreatment staging was done using TNM staging system. Stage IV was most common due to T4 disease, i.e. $62.9 \%(n=51)$. Radiologically regional nodes were positive in $33.3 \%(n=27)$ cases. Node negativity was observed in $58.0 \%$ ( $n=47, p<0.001)$; whereas, in $8.64 \%$ $(n=7)$ patients nodal status could not be assessed (Table I). Twenty patients out of $46(43.5 \%)$ with SCC were having nodal metastasis. Patients with SCC mostly presented in stage IV and later than 6 months after the onset of symptoms.

Definitive surgery was done in $51.8 \%(n=42)$ patients; remaining $(n=39)$ were treated with chemotherapy, radiotherapy or chemoradiotherapy. Two patients $(2.47 \%)$ were treated with chemotherapy alone, 24 $(29.62 \%)$ were treated with RT alone, and 13 (16.04\%) received CRT. Surgery and RT was given to 30 $(37.03 \%)$. Surgery with CRT was the treatment modality in $12(14.81 \%)$ patients. However, in patients with SCC, surgery was performed in only 13 out of 46 patients $(28.3 \%)$.

Survival outcomes were calculated for the patients with SCC. All patients were ECOG 2 or under and thus offered treatment. Median overall survival (OS) was 34.00 months (SE, $1.84,95 \% \mathrm{Cl}, 30.38-37.61$ ). Forty deaths were reported during the study period, and 6 were alive at the time of data collection. Median diseasefree survival (DFS) was 19.00 months (SE, 1.65, 95\% $\mathrm{Cl}, 15.75-22.25)$. All patients had experienced recurrence during the follow-up period.

Survival outcomes were compared with TNM, stage, and gender. No significant difference in overall survival of patients with different $T$ stage was found $(p=0.33)$.

Nodal status had significant impact on treatment outcomes. Patients with NO status had significantly better OS (Log-rank sig. $p<0.001$ ) when compared with the patient population with node positivity. Median OS in patients without nodal involvement $(\mathrm{N} 0, \mathrm{n}=24)$ remained 42.00 months (SE: $4.35,95 \% \mathrm{Cl}, 33.46-50.53$ ). In N +ve patients $(n=21)$, median OS was 30.00 (SE: $8.57,95 \%$ $\mathrm{Cl}, 13.81-46.18$ ) months.

Patients with non-metastasised disease $(n=42)$ had median OS of 36.00 months (SE: $2.070,95 \% \mathrm{Cl}$, 31.9440.05). In patients with metastases $(n=2)$, OS remained 
Table I: Patient characteristics, presenting symptoms and risk factors observed in patient population.

\begin{tabular}{|c|c|c|}
\hline Patient characteristics & Frequency & Percentage \\
\hline \multicolumn{3}{|l|}{ Gender distribution } \\
\hline Male & 51 & $62 \%$ \\
\hline Female & 30 & $38 \%$ \\
\hline \multicolumn{3}{|c|}{ Median age 50.00 years (IQR: $60.75-40.00$ ) } \\
\hline \multicolumn{3}{|l|}{ Histopathology } \\
\hline Squamous cell & 46 & $56.7 \%$ \\
\hline Adenoid cystic CA & 8 & $9.9 \%$ \\
\hline Adenocarcinoma & 7 & $8.6 \%$ \\
\hline Melanoma & 3 & $3.7 \%$ \\
\hline Rhabdomyosarcoma & 3 & $3.7 \%$ \\
\hline Plasmacytoma & 2 & $2.4 \%$ \\
\hline Non-Hodgkin's Lymphoma & 2 & $2.4 \%$ \\
\hline Papillary carcinoma & 2 & $2.4 \%$ \\
\hline Papillary adenocarcinoma & 2 & $2.4 \%$ \\
\hline Giant cell Sarcoma & 1 & $1.2 \%$ \\
\hline Spindle cell carcinoma & 1 & $1.2 \%$ \\
\hline Sarcomatoid carcinoma & 1 & $1.2 \%$ \\
\hline Ewing sarcoma & 1 & $1.2 \%$ \\
\hline Undifferentiated carcinoma & 2 & $2.4 \%$ \\
\hline \multicolumn{3}{|l|}{ Primary tumor site } \\
\hline Nasal cavity & 35 & $44 \%$ \\
\hline Maxillary sinus & 41 & $51 \%$ \\
\hline Other sinuses & 5 & $5 \%$ \\
\hline \multicolumn{3}{|l|}{ Stage-wise distribution } \\
\hline I & 2 & $2.4 \%$ \\
\hline II & 12 & $15 \%$ \\
\hline III & 13 & $16 \%$ \\
\hline IV & 51 & $62 \%$ \\
\hline Unknown & 3 & $3.7 \%$ \\
\hline \multicolumn{3}{|l|}{ Nodal status } \\
\hline No & 47 & $58.0 \%$ \\
\hline N1 & 15 & $18.5 \%$ \\
\hline N2 & 8 & $9.87 \%$ \\
\hline N3 & 4 & $4.93 \%$ \\
\hline $\mathrm{Nx}$ & 7 & $8.64 \%$ \\
\hline \multicolumn{3}{|l|}{ Presenting symptoms } \\
\hline Nasal blockage & 36 & $45 \%$ \\
\hline Headache & 32 & $40 \%$ \\
\hline Nasal bleeding & 24 & $30 \%$ \\
\hline Mass & 20 & $25 \%$ \\
\hline Pain & 16 & $20 \%$ \\
\hline Swelling of face & 14 & $18 \%$ \\
\hline Proptosis & 6 & $8 \%$ \\
\hline Dysphagia & 1 & $2 \%$ \\
\hline Loss of vision & 6 & $8 \%$ \\
\hline Tooth eruption & 1 & $3 \%$ \\
\hline \multicolumn{3}{|l|}{ Risk factors } \\
\hline Cigarette smoking & 19 & $23.4 \%$ \\
\hline Occupational & 13 & $16.0 \%$ \\
\hline Chronic sinusitis & 9 & $11.1 \%$ \\
\hline Huqqa smoker & 7 & $8.6 \%$ \\
\hline Niswar/pan & 5 & $6.2 \%$ \\
\hline Alcohol & 2 & $2.5 \%$ \\
\hline Insignificant & 26 & $32.1 \%$ \\
\hline
\end{tabular}

8.00 months only; SE and $95 \% \mathrm{Cl}$ could not be computed due to the small number. In two patients, status of distant metastases was unknown. Value of Log-rank (MantelCox) was significant $(p=0.001)$.

Different treatment modalities were compared in terms of treatment outcomes. Patients receiving RT had significantly better OS when compared with having no RT $(p=0.001)$. Patients receiving no RT $(n=10)$ had median OS of 13.00 months (SE: $9.49,95 \% \mathrm{Cl}, 0.00-$ $31.59)$ while those receiving RT $(n=36)$ had significantly better survival being 38.00 months (SE: $5.29,95 \% \mathrm{Cl}$, 27.63-48.36).

Patients with and without surgery had no significant difference in median OS $(p=0.278)$. However, patients who had undergone surgery $(n=13)$ had slightly better median OS 38.00 months (SE: 14.60, 95\% Cl, 9.39$66.61)$ vs. 34.00 (SE: 1.95, 30.17-37.82) in patients with no surgical treatment $(n=33)$ although statistical significance could not be achieved.

Chemotherapy was also not a statistically significant factor in survival. Survival outcomes in chemotherapy vs. no chemotherapy were 42.00 months (SE: $4.18,95 \%$ $\mathrm{Cl}, 33.80-50.20$ ) and 33.00 (SE: 1.31, 95\% Cl, 30.4235.58). Chemotherapy had positive impact on survival outcome increasing OS from 33.00 months to 42.00 months but without statistical significance $(p=0.951)$.

Patients receiving CRT $(n=9)$ had median OS of 23.00 months (SE: $7.45,95 \% \mathrm{Cl}, 8.39$ - 37.60) in comparison with patients receiving no CRT $(\mathrm{n}=37)$ i.e. 36.00 months (SE: $2.29,95 \% \mathrm{Cl}, 31.49-40.50$ ). Patients without CRT had better median OS but not statistically significant $(p=0.390)$.

Stage-wise survival outcomes were compared applying Log-rank (Mantel-Cox) survival comparison. Significantly different survival outcomes $(p=0.014)$ were observed in different stages (Table II). Maximum patients were enrolled in stage IVB $(n=13)$ followed by IVA $(n=11)$, and only two patients were enrolled with IVC having squamous cell histology. Maximum survival of 51.00 months (SE: $2.739,95 \% \mathrm{Cl}$, 45.63-56.37) was achieved in stage III and minimum of 8.00 in stage IVC (Table II).

Six patients out of 46 could not complete first year of treatment. Forty $(87 \%)$ were alive after first year. The cumulative survival at 1,2 , and 3 years was $87 \%, 48 \%$ and $29 \%$, respectively with median being 2.83 years.

\section{DISCUSSION}

Nasal and paranasal cancer, being rare, is not included specifically in Globocan data, so incidence is usually measured from individual institutional studies and metaanalyses. In the last 15 years, 81 patients were enrolled at the study place $(0.2 \%$ hospital-based incidence) which is in accordance with global statistics showing incidence of $0.2-0.8 \%$ of all malignancies worldwide. ${ }^{7}$ Most of the 
Table II: Stage-wise overall survival (months) in patients with squamous cell carcinoma.

\begin{tabular}{l|c|c|c|c|c|c|c}
\hline Stage & Total patients & Deaths & Alive* & Median OS & Standard error & \multicolumn{2}{|c}{$95 \%$ Confidence interval } \\
\cline { 5 - 7 } & & & & & & Lower bound & Upper bound \\
\hline II & 6 & 5 & 1 & 33.00 & 14.00 & 5.56 & 60.44 \\
III & 8 & 8 & 0 & 51.00 & 2.73 & 45.63 \\
IV/Unspecified & 6 & 4 & 2 & 36.00 & 7.85 & 20.60 \\
IVA & 11 & 10 & 1 & 31.00 & 13.15 & 5.22 & 51.39 \\
IVB & 13 & 11 & 2 & 34.00 & 0.948 & 32.14 \\
IVC & 2 & 2 & 0 & 8.00 & - & - & 35.85 \\
Overall & 46 & 40 & 6 & 34.00 & 1.84 & 30.38 & 37.61 \\
\hline
\end{tabular}

${ }^{*}$ Alive at the time of study.
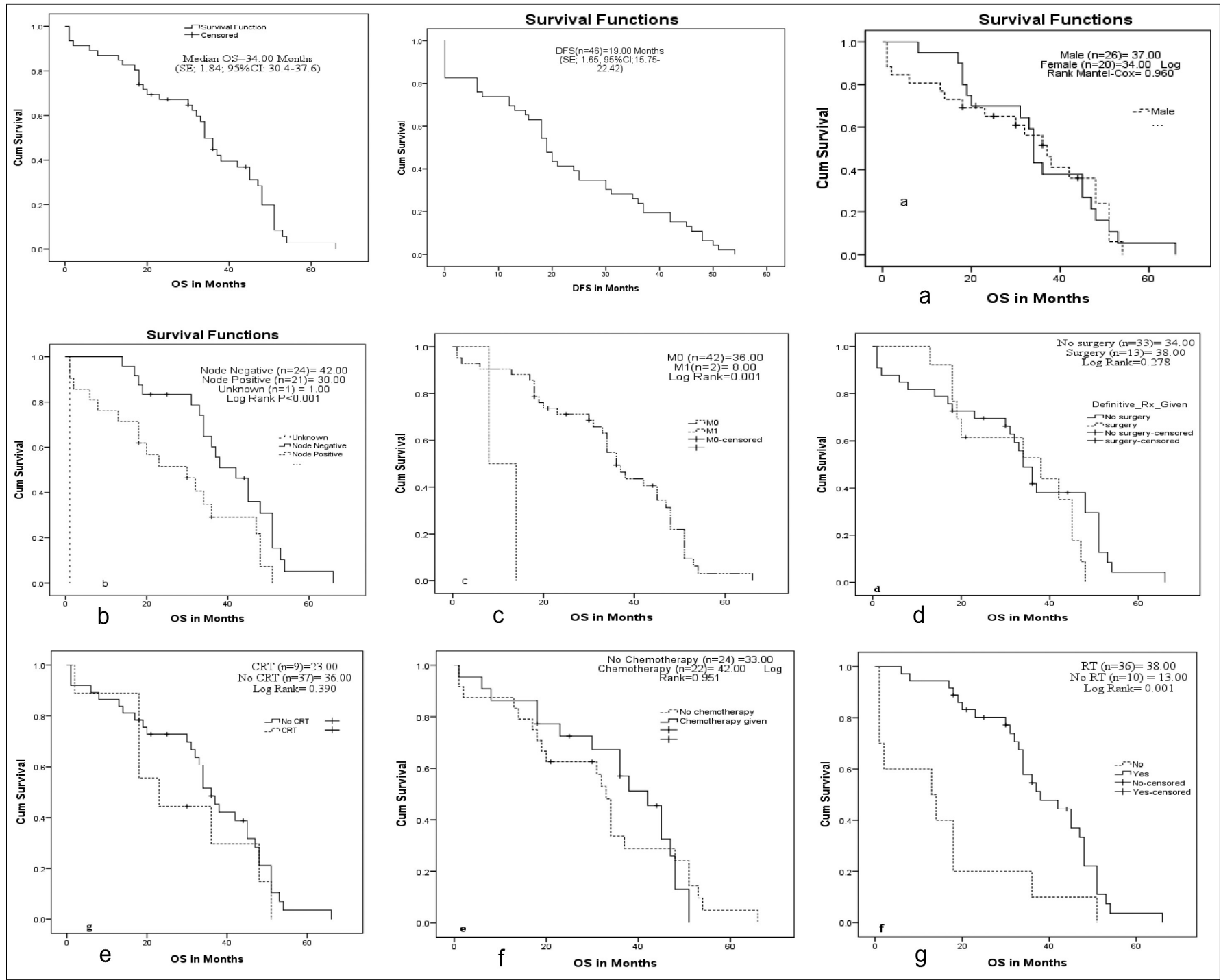

Figure 1: Kaplan Meier curve showing overall survival, disease-free survival, and effects of (a) Gender (b) Nodal involvement (c) Metastatic status (d) Surgery (e) Chemotherapy (f) RT, and (g) CRT on overall survival.

reported studies are single institution retrospective analyses; thus wide range of treatment outcomes are also reported. 8 In this study, maxillary sinus $(51 \% ; n=41)$ and nasal cavity $(44 \% ; n=35)$ were the most commonly occurring primary sites, while the ethmoid, sphenoid and frontal sinuses constituted the rare ( $5 \%$ of the tumors). Analogous disease patterns are observed in literature. ${ }^{9}$ Patients are often asymptomatic until late in the course of disease, i.e. $77 \%$ patients presented with stage III and IV in this study. Most of patients had latent period of presentation of more than 6 months $(n=39)$ and the time to presentation of 3 to 6 months $(n=32)$. One reason for late presentation besides indolent course of disease was being initially misdiagnosed as sinusitis.

As compared to other head and neck tumors, nodal metastasis in SNM is less commonly seen i.e. $66 \%$ of 
patients were node negative. This is consistent with global statistics which show that nodal and distant metastasis are rare features in case of nasal and PNS tumors. 10

In this study, squamous cell carcinoma was the most common histopathology among all patients (56.7\%, $\mathrm{n}=46$ ). Previously reported pattern for Pakistani population has stated squamous cell carcinoma in $44 \%$, adenoid cystic carcinoma in $25 \%$, and mucoepidermoid carcinoma in $15 \%$ patients. ${ }^{11}$

In this study population, no significant survival benefits were observed with or without adjuvant chemotherapy and chemo-radiotherapy. It is in concordance with literature. 12 Radiotherapy was the only significant factor in improving overall survival. Survival outcomes with and without RT remained 3.16 vs. 1.08 with statistical significance. Out of 46 patients of squamous cell histology, 18 had received RT alone and most of them were in advanced stage. Patients receiving doses more than $66 \mathrm{~Gy}$ had more complications while local control was similar which may be due to less number of individuals being studied or suboptimal contouring of CTV as patients receiving doses in excess of $66 \mathrm{~Gy}$ were planned using 3D conformal treatment planning. Local recurrence is major cause of treatment failure as compared to metastatic disease in sinonasal malignancies. Recurrence was observed in all patients within 3 years, while distant metastasis was observed in two patients only with squamous cell carcinoma. Median disease-free interval was observed to be 19.00 months (1.60 years; range of 1.30-1.89 years; Figure 1). Gender and $T$ stage were of no significant prognostic value, while disease stage had significant effect on survival outcomes.

As other observational hospital-based studies, our work has limitations and strengths. A major limitation is related to the sample size; it would be desirable to analyse a larger patient number in order to have enough statistical power for certain results. Although multiple histopathologies were observed while dealing with nasal and paranasal sinus tumors, their frequencies were too low for reasonable analysis and so treatment outcome was limited to squamous cell histology.

Although it is not possible to avoid completely any bias, we think that results were not chance findings. Anyway, generalisability is limited due to the population features: which mostly belong to low socioeconomic strata.

\section{CONCLUSION}

SNM comprised of $0.2 \%$ of malignancies presenting to our Institute with maxillary sinus malignancies accounting for half of the cases with a slight male predominance (1.7:1). Nasal blockage along with headache being the most common presenting complaint. SCC is the most common histology seen in SNMs. Two-thirds of the patients present with locally advanced disease (Stage III, IVA and IVB). Median age is 50 years with smoking as the chief risk factor. Majority of cases are diagnosed at advanced stage and receive multimodality treatment. RT in both adjuvant and definitive setting has a survival advantage, while CCRT appeared to be producing a significant decline in median survival. Most important factors influencing survival were nodal involvement, distant metastases, and stage at presentation. Sharpest gradient in median OS was seen with increase in stage at presentation, ranging from 51 months in stage III to 8 months in stage IVC.

\section{REFERENCES}

1. Mahalingappa YB, Khalil HS. Sinonasal malignancy: presentation and outcomes. J Laryngol Otol 2014; 128:654-7.

2. Pfister DG, Ang KK, Brizel DM, Burtness BA, Busse PM, Caudell JJ, et al. Head and neck cancers, version 2. 2013. Featured updates to the NCCN guidelines. J Natl Compr Canc Netw 2013; 11:917-23.

3. Ansa B, Goodman M, Ward K, Kono SA, Owonikoko TK, Higgins $\mathrm{K}$, et al. Paranasal sinus squamous cell carcinoma incidence and survival based on surveillance, epidemiology, and end results data, 1973 to 2009. Cancer 2013; 119:2602-10.

4. Llorente JL, López F, Suárez C, Hermsen MA. Sinonasal carcinoma: Clinical, pathological, genetic and therapeutic advances. Nat Rev Clin Oncol 2014; 11:460-72.

5. Poursadegh M, Poursadegh F, Esmaeili M, Bakhshaee M. Epidemiological survey of sinonasal Malignancy in North-East Iran. Iran J Otorhinolaryngol 2015; 27:225-9.

6. Sanghvi S, Khan MN, Patel NR, Yeldand S, Baredes S, Eloy JA. Epidemiology of sinonasal squamous cell carcinoma: A comprehensive analysis of 4994 patients. Laryngoscope $2014 ;$ 124:76-83

7. Youlden DR, Cramb SM, Peters S, Porceddu SV, Møller H, Fritschi L, et al. International comparisons of the incidence and mortality of sinonasal cancer. Cancer Epidemiol 2013; 37:770-9.

8. Birgi SD, Teo M, Dyker KE, Sen M, Prestwich RJD. Definitive and adjuvant radiotherapy for sinonasal squamous cell carcinomas: A single institutional experience. Radiat Oncol 2015; 10:190.

9. Shirazi N, Bist SS, Selvi TN, Harsh M. Spectrum of sinonasal tumors: A 10-year experience at a tertiary care hospital in North India. Oman Med J 2015; 30:435-40.

10. Guan X, Wang X, Liu Y, Hu C, Zhu G. Lymph node metastasis in sinonasal squamous cell carcinoma treated with IMRT/3DCRT. Oral Oncol 2013; 49:60-5

11. Kazi M, Awan S, Junaid M, Qadeer S, Hassan NH. Management of sinonasal tumors: Prognostic factors and outcomes: A 10-year experience at a tertiary care hospital. Indian J Otolaryngol Head Neck Surg 2013; 65(Suppl 1):155-9.

12. Syrjänen $K$, Syrjänen $S$. Detection of human papilloma virus in sinonasal papillomas: Systematic review and meta-analysis. Laryngoscope 2013; 123:181-92.

.......... 